\title{
Efficacy and safety of allergen immunotherapy for IgE-mediated food allergy: systematic review and meta-analysis
}

Debra de Silva ${ }^{1}$, Pablo Rodriguez del Rio ${ }^{2}$, Nicolette w Jong ${ }^{3}$, Ekaterina Khaleva ${ }^{4}$, C. Singh $^{5}$, Anna Nowak-Wegrzyn ${ }^{6}$, Antonella Muraro ${ }^{7}$, Philippe Bégin ${ }^{8}$, Giovanni Pajno ${ }^{9}$, Alessandro Fiocchi ${ }^{10}$, Angel Sanchez San ${ }^{11}$, Carla Jones ${ }^{12}$, Caroline Nilsson ${ }^{13}$, Carsten Bindslev-Jensen ${ }^{14}$, Gary Wong ${ }^{15}$, Hugh Sampson ${ }^{16}$, Kirsten Beyer ${ }^{17}$, Mary Jane Marchisotto $^{18}$, Montserrat Fernandez-Rivas ${ }^{19}$, Rosan Meyer $^{20}$, Susanne Lau ${ }^{17}$, Ulugbek Nurmatov $^{21}$, and Graham Roberts ${ }^{4}$

${ }^{1}$ The Evidence Centre England

${ }^{2}$ Hospital Infantil Universitario Nino Jesus

${ }^{3}$ University Medical Centre Rotterdam the Netherlands

${ }^{4}$ University of Southampton Faculty of Medicine

${ }^{5}$ The Evidence Centre New Zealand

${ }^{6}$ NYU Langone Health

${ }^{7}$ Padua University Hospital Italy

${ }^{8}$ Univerisite de Montreal Canada

${ }^{9}$ Policlinico Hospital-University of Messina Italy

${ }^{10}$ Ospedale Pediatrico Bambino Gesu

${ }^{11}$ AEPNAA Spanish Association for People with Food and Latex Allergy Spain

${ }^{12}$ Allergy UK England

${ }^{13}$ Karolinska Institutet and Sachs' Children and Youth Hospital Sweden

${ }^{14}$ Odense Universitetshospital

${ }^{15}$ Chinese University of Hong Kong Hong Kong

${ }^{16}$ Icahn School of Medicine at Mount Sinai Department of Medicine

${ }^{17}$ Charite Universitatsmedizin Berlin

${ }^{18}$ European Academy of Allergy and Clinical Immunology

${ }^{19}$ Hospital Clínico San Carlos Spain

${ }^{20}$ Imperial College London

${ }^{21}$ Cardiff University

November 15, 2021

\footnotetext{
Abstract

Background There is substantial interest in allergen-specific immunotherapy in food allergy. We systematically reviewed its efficacy and safety. Methods We searched six bibliographic databases from 1946 to 30 April 2021 for randomised controlled trials about immunotherapy alone or with biologicals in IgE-mediated food allergy confirmed by oral food challenge. We pooled the data using random-effects meta-analysis. Results We included 36 trials with 2,126 participants, mainly children. Oral immunotherapy increased tolerance whilst on therapy for peanut (RR 9.9, 95\% CI 4.5. to 21.4, high certainty); cow's milk (RR 5.7, 1.9 to 16.7 , moderate certainty) and hen's egg allergy (RR 8.9, 4.4 to 18 , moderate certainty). The number needed to treat
} 
to increase tolerance to a single dose of $300 \mathrm{mg}$ or $1000 \mathrm{mg}$ peanut protein was 2 . In peanut allergy, oral immunotherapy did not increase adverse reactions ( $R R$ 1.1, 1.0 to 1.2, low certainty) or severe reactions (RR 1,6, 0.7 to 3.5, low certainty). It may increase adverse reactions in cow's milk (RR 3.9, 2.1 to 7.5, low certainty) and hen's egg allergy (RR 7.0, 2.4 to 19.8, moderate certainty), but reactions tended to be mild and gastrointestinal. Epicutaneous immunotherapy increased tolerance whilst on therapy for peanut (RR 2.6, 1.8 to 3.8, moderate certainty). Results were unclear for other allergies and administration routes. Conclusions Oral immunotherapy improves tolerance whilst on therapy and is probably safe in peanut, cow's milk and hen's egg allergy. However, our review found little about whether this improves quality of life, is sustained or cost-effective.

\section{Hosted file}

AIT systematic review (for submission).docx available at https://authorea.com/users/333888/ articles/545579-efficacy-and-safety-of-allergen-immunotherapy-for-ige-mediated-foodallergy-systematic-review-and-meta-analysis

\section{Hosted file}

Figure 1.doc available at https://authorea.com/users/333888/articles/545579-efficacy-andsafety-of-allergen-immunotherapy-for-ige-mediated-food-allergy-systematic-review-andmeta-analysis 